John M. Kirkwood, M.D.

Hillman UPMC Cancer Center

Pittsburgh, PA

Since publication of their article, the authors report no further potential conflict of interest.

1. Faries MB, Thompson JF, Cochran AJ, et al. Completion dissection or observation for sentinel-node metastasis in melanoma. N Engl J Med 2017;376:2211-22.

2. Azimi F, Scolyer RA, Rumcheva P, et al. Tumor-infiltrating lymphocyte grade is an independent predictor of sentinel lymph node status and survival in patients with cutaneous melanoma. J Clin Oncol 2012;30:2678-83.

3. Morton DL, Thompson JF, Cochran AJ, et al. Sentinel-node biopsy or nodal observation in melanoma. N Engl J Med 2006; 355:1307-17.

DOI: 10.1056/NEJMc1716071

DR. WEBER AND COLLEAGUeS REPLY: The basis of the queries by Thomson et al. regarding regularly performed surveillance scans is associated with broader efforts to more effectively identify relapses in high-risk melanoma. ${ }^{1}$ In our trial, data on disease symptoms were not collected at the time of the scans and would not have been recorded as adverse events if they had occurred more than 100 days after treatment. Radiographic versus symptomatic data were not available, but among the patients who received subsequent anticancer therapy (129 patients [28.5\%] in the nivolumab group and 171 patients [37.7\%] in the ipilimumab group), 24 patients in each group (5.3\% of the trial population) underwent surgery with curative intent.

In response to Maria et al.: we provide further context on the protocol amendment that established the interim analysis. Trial recruitment was rapid. Approximately 900 patients underwent randomization in a double-blind fashion, with 800 patients planned for a time-driven (rather than event-driven) final analysis of recurrencefree survival at a minimum of 36 months of follow-up. The protocol amendment was based on ongoing review of pooled, blinded data, including data on rates of recurrence-free survival and overall survival. The originally anticipated target of 507 events of recurrence-free survival was revised to 450 for the final analysis on the basis of patient distribution according to AJCC disease stage, a slower event rate, a higher cure rate, and a higher rate of early withdrawal from the trial. We determined that 450 events would provide a power of $85 \%$ to detect a hazard ratio for disease recurrence or death of 0.75 (under the 0.83 cutoff for significance) with an overall two-sided type I error rate of 0.05 .

In the interim analysis, 360 of the 450 events $(80 \%)$ had occurred, and before the database lock, amendment 18 specifying an interim analysis was implemented on January 26, 2017. The stopping boundary was derived on the basis of the 360 events with the use of a Lan-DeMets alpha spending function with O'Brien-Fleming boundaries, with a critical hazard ratio of 0.78 (adjusted two-sided alpha level of 0.0244). An independent data and safety monitoring committee met on June 30, 2017, to review the formal interim analysis of recurrence-free survival (database lock, June 12, 2017). The committee confirmed that the prespecified boundary for significance was crossed (hazard ratio for recurrence-free survival, 0.65 ; $95.56 \%$ confidence interval, 0.51 to $0.83 ; \mathrm{P}<0.001)$, with no new safety signals identified.

Jeffrey Weber, M.D., Ph.D.

New York University Perlmutter Cancer Center New York, NY

jeffrey.weber2@nyumc.org

Anila Qureshi, M.D., M.P.H.

Bristol-Myers Squibb

Princeton, NJ

Paolo A. Ascierto, M.D.

Istituto Nazionale Tumori Fondazione Pascale

Naples, Italy

Since publication of their article, the authors report no further potential conflict of interest.

1. Romano E, Scordo M, Dusza SW, Coit DG, Chapman PB. Site and timing of first relapse in stage III melanoma patients: implications for follow-up guidelines. J Clin Oncol 2010;28:3042-7.

DOI: 10.1056/NEJMc1716071

\title{
Mepolizumab for Eosinophilic COPD
}

TO THE EDITOR: Pavord and colleagues (Oct. 26 issue $)^{1}$ report that treatment with mepolizumab resulted in a lower annual rate of exacerbations than placebo among patients with eosinophilic chronic obstructive pulmonary disease (COPD). The trial enrolled patients who had a documented diagnosis of COPD with an eosinophilic phenotype, thus a portion of these patients might be 
characterized as having the asthma-COPD overlap syndrome (ACOS). The clinical features, including persistent airflow limitation, smoking history, elevated blood eosinophil count, use of high-dose glucocorticoid therapy, and frequent exacerbations, meet the criteria set for ACOS by the Global Initiative for Asthma (http://ginasthma .org). Moreover, the therapeutic effect of mepolizumab in patients with COPD was associated with eosinophil counts. Previous research has shown that mepolizumab mitigated severe eosinophilic asthma in patients who also had features of COPD. ${ }^{2,3}$ These findings suggest that patients with ACOS could benefit from anti-interleukin-5 treatment. Similar observations were reported in studies of dupilumab (anti-interleukin-4) and omalizumab (anti-IgE) in patients with ACOS.,5

Yang Xia, M.D., Ph.D.

Wen Li, M.D.

Huahao Shen, M.D.

Second Affiliated Hospital of Zhejiang University School of Medicine

Hangzhou, China

huahaoshen@zju.edu.cn

No potential conflict of interest relevant to this letter was reported.

1. Pavord ID, Chanez P, Criner GJ, et al. Mepolizumab for eosinophilic chronic obstructive pulmonary disease. N Engl J Med 2017;377:1613-29.

2. Ortega HG, Liu MC, Pavord ID, et al. Mepolizumab treatment in patients with severe eosinophilic asthma. N Engl J Med 2014;371:1198-207.

3. Yancey S, Albers FC, Gunsoy N, Harris S, Keene O. Effect of mepolizumab on exacerbations in asthma patients with features common in COPD. Am J Respir Crit Care Med 2017;195:A4683. abstract.

4. Wenzel SE, Jayawardena S, Graham NM, Pirozzi G, Teper A. Severe asthma and asthma-chronic obstructive pulmonary disease syndrome - authors' reply. Lancet 2016;388:2742.

5. Maltby S, Gibson PG, Powell H, McDonald VM. Omalizumab treatment response in a population with severe allergic asthma and overlapping COPD. Chest 2017;151:78-89.

DOI: 10.1056/NEJMc1715454

TO THE EDITOR: Pavord et al. report the findings of trials evaluating the effects of mepolizumab in patients with an eosinophilic phenotype of COPD. We offer three reasons that the blood eosinophil count may be inferior to the sputum eosinophil count as a biomarker in these patients.

First, various diseases, including allergy and atopy, may also affect the blood eosinophil count. Second, studies have shown disparities regarding the sensitivity and specificity of the blood eosinophil count to identify sputum eosinophil counts at levels greater than $3 \% .^{1,2}$ Third, previous work suggests that the blood eosinophil count may not be a generalizable biomarker to define clinical COPD phenotypes. ${ }^{3}$ The results of a combined analysis of SPIROMICS (Subpopulations and Intermediate Outcomes Measures in COPD Study) and the COPDGene (Genetic Epidemiology of COPD) study also did not show a robust association between blood eosinophil count and the occurrence of exacerbations. ${ }^{4}$ Do the authors think that additional trials that are based on sputum eosinophil count will yield more clinically directive results?

\section{Parichehr Shahlavi-Monavvar, Pharm.D.}

Tabriz University of Medical Sciences

Tabriz, Iran

Abdolkarim Mobasher-Jannat, M.D.

Baqiyatallah University of Medical Sciences

Tehran, Iran

karim.jannat@yahoo.com

No potential conflict of interest relevant to this letter was reported.

1. Bafadhel M, McKenna S, Terry S, et al. Acute exacerbations of chronic obstructive pulmonary disease: identification of biologic clusters and their biomarkers. Am J Respir Crit Care Med 2011;184:662-71.

2. Negewo NA, McDonald VM, Baines KJ, et al. Peripheral blood eosinophils: a surrogate marker for airway eosinophilia in stable COPD. Int J Chron Obstruct Pulmon Dis 2016;11:1495504.

3. Zysman M, Deslee G, Caillaud D, et al. Relationship between blood eosinophils, clinical characteristics, and mortality in patients with COPD. Int J Chron Obstruct Pulmon Dis 2017;12: 1819-24.

4. Keene JD, Jacobson S, Kechris K, et al. Biomarkers predictive of exacerbations in the SPIROMICS and COPDGene cohorts. Am J Respir Crit Care Med 2017;195:473-81.

DOI: $10.1056 / N E J M c 1715454$

THE AUTHORS REPLY: Xia et al. suggest that some of our patients may have had ACOS and that this population may benefit specifically from antiinterleukin-5 treatment. The ACOS classification attempts to define a subgroup of patients who have features that are classically associated with both COPD and asthma, including the degree of airflow limitation, lung-function variability, blood eosinophil count, history of atopy, and smoking history (http://goldcopd.org). In fact, these traits are inconsistently present in most patients with COPD and represent a continuum of traits that may have little or no association with each other. ${ }^{1}$

Moreover, the geometric mean blood eosinophil count is similar in populations of patients with COPD and in those with asthma, and recent large-scale analyses show no correlation between 


\begin{tabular}{|c|c|c|c|c|}
\hline \multirow[t]{2}{*}{ FEV $_{1}$ Reversibility } & \multicolumn{2}{|c|}{ METREX } & \multirow{2}{*}{$\begin{array}{l}\text { METREO } \\
\text { All Patients } \\
(\mathrm{N}=674) \dagger\end{array}$} & \multirow{2}{*}{$\begin{array}{c}\text { Both Trials } \\
\text { Patients with } \\
\text { Eosinophilic Phenotype } \\
(\mathrm{N}=1136)\end{array}$} \\
\hline & $\begin{array}{c}\text { Patients with } \\
\text { Eosinophilic Phenotype } \\
(\mathrm{N}=462)\end{array}$ & $\begin{array}{l}\text { All Patients } \\
(\mathrm{N}=836)\end{array}$ & & \\
\hline \multicolumn{5}{|l|}{ Yes } \\
\hline No. of patients (\%) & $71(15)$ & $121(14)$ & $108(16)$ & $179(16)$ \\
\hline Blood eosinophil count - cells $/ \mathrm{mm}^{3} \uparrow$ & $290 \pm 0.609$ & $160 \pm 0.959$ & $220 \pm 0.809$ & $250 \pm 0.746$ \\
\hline \multicolumn{5}{|l|}{ No } \\
\hline No. of patients (\%) & $391(85)$ & $715(86)$ & $565(84)$ & $956(84)$ \\
\hline Blood eosinophil count - cells $/ \mathrm{mm}^{3}+$ & $260 \pm 0.566$ & $140 \pm 0.943$ & $230 \pm 0.854$ & $240 \pm 0.752$ \\
\hline
\end{tabular}

* Plus-minus values are geometric means $\pm S D$ on a $\log _{\mathrm{E}}$ scale. Data are from the modified intention-to-treat populations of patients with chronic obstructive pulmonary disease (COPD) in the METREX (Mepolizumab vs. Placebo as Add-on Treatment for Frequently Exacerbating COPD Patients) and METREO (Mepolizumab vs. Placebo as Add-on Treatment for Frequently Exacerbating COPD Patients Characterized by Eosinophil Level) trials; all the patients in these trials received mepolizumab or placebo. Reversibility was defined as an increase of at least $12 \%$ and at least $200 \mathrm{ml}$ in the forced expiratory volume in 1 second $\left(\mathrm{FEV}_{1}\right)$ after bronchodilator use, on the basis of the reversibility criteria from the Global Initiative for COPD for patients with the asthma-COPD overlap syndrome (http://goldcopd.org).

$\uparrow$ Reversibility was not assessed for one participant in the METREO trial because a measure of the $\mathrm{FEV}_{1}$ before bronchodilator use was not obtained at screening.

$¥$ The blood eosinophil count was ascertained at enrollment.

eosinophil counts and the degree of reversibility in the forced expiratory volume in 1 second $\left(\mathrm{FEV}_{1}\right)$ after bronchodilator use, smoking status, or atopy. ${ }^{2}$ In a post hoc analysis involving patients in the METREX (Mepolizumab vs. Placebo as Add-on Treatment for Frequently Exacerbating COPD Patients) and METREO (Mepolizumab vs. Placebo as Add-on Treatment for Frequently Exacerbating COPD Patients Characterized by Eosinophil Level) trials, we found no relationship between $\mathrm{FEV}_{1}$ reversibility after bronchodilator use and blood eosinophil counts (Table 1). We suggest that rather than defining unique disease subtypes such as ACOS, the classification of disease and selection of therapy would be better directed by a precision approach that targets independent mechanisms driving the disease (treatable traits) ${ }^{1}$, which often vary independently within an individual patient.

Shahlavi-Monavvar and Mobasher-Jannat suggest that the sputum eosinophil count is superior to the blood eosinophil count as a biomarker for the prediction of response to mepolizumab. There are two points to consider. First, the recent study conducted by Kolsum et al. ${ }^{3}$ showed that blood and sputum eosinophil counts are positively associated; therefore, if sputum is a superior biomarker, then blood counts could be an equivalent and more convenient substitute. Second, and more importantly, in the DREAM
(Dose Ranging Efficacy and Safety with Mepolizumab in Severe Asthma) study involving patients with severe eosinophilic asthma, the sputum eosinophil count was shown to be less predictive of response to mepolizumab than the blood eosinophil count. ${ }^{4}$ In contrast to the sputum eosinophil count, the blood eosinophil count represents a readily accessible biomarker that is already routinely measured in most patients with COPD. ${ }^{5}$ Our study provides additional evidence that the blood eosinophil count can be used to identify patients with COPD who may benefit from therapies targeting eosinophilic inflammation. ${ }^{2,5}$

Frank C. Sciurba, M.D.

University of Pittsburgh

Pittsburgh, PA

sciurbafc@upmc.edu

Eric S. Bradford, M.D.

GlaxoSmithKline

Research Triangle Park, NC

Ian D. Pavord, D.M.

Oxford Respiratory NIHR Biomedical Research Centre

Oxford, United Kingdom

Since publication of their article, the authors report no further potential conflict of interest.

1. Agusti A, Bel E, Thomas M, et al. Treatable traits: toward precision medicine of chronic airway diseases. Eur Respir J 2016; 47:410-9.

2. Pavord ID, Lettis S, Locantore N, et al. Blood eosinophils and inhaled corticosteroid/long-acting $\beta$-2 agonist efficacy in COPD. Thorax 2016;71:118-25. 
3. Kolsum U, Damera G, Pham TH, et al. Pulmonary inflammation in patients with chronic obstructive pulmonary disease with higher blood eosinophil counts. J Allergy Clin Immunol 2017;140(4):1181-1184.e7.

4. Pavord ID, Korn S, Howarth P, et al. Mepolizumab for severe eosinophilic asthma (DREAM): a multicentre, double-blind, placebo-controlled trial. Lancet 2012;380:651-9.

5. Bafadhel M. Eosinophils in COPD: are we nearly there yet? Lancet Respir Med 2017;5:913-4.

DOI: $10.1056 / N E J M c 1715454$

\section{Diuretic Treatment in Heart Failure}

TO THE EDITOR: The review article by Ellison and Felker (Nov. 16 issue) ${ }^{1}$ has a two-edged potential. Physicians are encouraged to use diuretics as first-line therapy for heart failure, but discontent with the drugs runs like a thread through the review. We had hoped that the section on other approaches and future directions would address how to minimize the use of diuretics. Long-term use of diuretic therapy for chronic heart failure may contribute to neurohumoral stimulation. ${ }^{2}$ Therefore, not only does skillful use of diuretic therapy remain fundamental to the successful management of heart failure, but so do strategies for their avoidance. First-line use of diuretics might influence adequate dosing of cardioprotective drugs, and the promotion of cardiac regeneration may become impractical. The latter is of particular importance the younger the patient with heart failure. ${ }^{3}$

\section{Dietmar Schranz, M.D., Ph.D.}

Pediatric Heart Center

Frankfurt, Germany

dietmar.schranz@kgu.de

No potential conflict of interest relevant to this letter was reported.

1. Ellison DH, Felker GM. Diuretic treatment in heart failure. N Engl J Med 2017;377:1964-75.

2. Schranz D, Voelkel NF. "Nihilism" of chronic heart failure therapy in children and why effective therapy is withheld. Eur J Pediatr 2016;175:445-55.

3. Mollova M, Bersell K, Walsh S, et al. Cardiomyocyte proliferation contributes to heart growth in young humans. Proc Natl Acad Sci U S A 2013;110:1446-51.

DOI: 10.1056/NEJMc1716477

TO THE EDITOR: In the sections on treatment of diuretic resistance and on adjuncts to diuretic therapy, the authors omit an important consideration. It has been reported that prolonged highdose treatment with furosemide can result in thiamine deficiency. ${ }^{1-3}$ Some studies have indicated that supplementation of thiamine in patients with heart failure improves cardiac function and urinary output. ${ }^{1,2,4}$ Administering thiamine to patients who receive continuing diuretic therapy is therefore in order. At least some patients may thus be spared the apparently paradoxical effect of "the more you give, the less you get."

Peter H. Berczeller, M.D.

New York University School of Medicine

New York, NY

peterberczeller@aol.com

No potential conflict of interest relevant to this letter was reported.

1. Katta N, Balla S, Alpert MA. Does long-term furosemide therapy cause thiamine deficiency in patients with heart failure? A focused review. Am J Med 2016;129(7):753.e7-753.e11.

2. Seligmann $\mathrm{H}$, Halkin $\mathrm{H}$, Rauchfleisch $\mathrm{S}$, et al. Thiamine deficiency in patients with congestive heart failure receiving longterm furosemide therapy: a pilot study. Am J Med 1991;91:151-5. 3. Misumida N, Umeda H, Iwase M. Shoshin beriberi induced by long-term administration of diuretics: a case report. Case Rep Cardiol 2014;2014:878915.

4. DiNicolantonio JJ, Niazi AK, Lavie CJ, O'Keefe JH, Ventura HO. Thiamine supplementation for the treatment of heart failure: a review of the literature. Congest Heart Fail 2013;19:214-22.

DOI: 10.1056/NEJMc1716477

TO THE EDITOR: Ellison and Felker report that the combination of hypertonic saline with high doses of loop diuretics has been proposed to improve renal dysfunction and natriuresis in congestive heart failure, although that approach has not been tested in robust trials. As reported by Gandhi et al., ${ }^{1}$ our group has conducted some trials on the role of high-dose furosemide and small-volume hypertonic saline solution infusion in the treatment of refractory congestive heart failure. The first, a randomized, single-blind study, ${ }^{2}$ showed that this treatment was associated with mortality reduction and clinical improvement during an extended follow-up period. A second study ${ }^{3}$ showed that patients who received hypertonic saline solution had a significantly faster reduction in brain natriuretic peptide levels and a lower incidence of readmission at follow-up than patients who did not receive it. A third study, ${ }^{4}$ which examined the effect of hypertonic saline solution on levels of natriuretic and immunoinflamma- 